\title{
Large File System Backup NERSC Global File System Experience
}

\author{
M. Andrews, J. Hick, W. Kramer, A. Mokhtarani \\ National Energy Research Scientific Computing Center at Lawrence Berkeley National \\ Laboratory
}

\begin{abstract}
NERSC's Global File system (NGF), accessible from all compute systems at NERSC, holds files and data from many scientific projects. A full backup of this file system to our High Performance Storage System (HPSS) is performed periodically. Disk storage usage by projects at NERSC has grown seven fold over a two year period, from $\sim 20 \mathrm{~TB}$ in June 2006 to $\sim 140$ TB in June 2008. The latest full backup took about 13 days and more than 200 T10k tape cartridges (.5 TB capacity). Petabyte file systems are becoming a reality in the next few years and the existing utilities are already strained in handling backup tasks.

In this report we investigate backup policies and tools for our High Performance Computing (HPC) center and will attempt to examine their strength and weaknesses. We will then examine the requirements for backup/restore in peta- and exa-scale environment and identify potential bottlenecks. Lastly, we will propose a list of desirable features and topologies for backing up petabyte-sized global file systems.

\section{Introduction}

Global file systems require significant increases in capacity each year to continue meeting ever-increasing data requirements from scientific applications. NERSC provides the NERSC Global File system (NGF), a large amount of on-line storage, to users for their daily storage needs. NGF is accessible from all compute systems at the center. Due to the importance of the global file system, the center has established a policy of providing backup of users' data with the goal of complete restoration of the file system to a known state in the event of a catastrophic failure. As the size of NGF increases to keep pace with user demand, NERSC should focus on the strategy it uses to perform the backups and ensure it will meet demand for years to come.
\end{abstract}

As of March 2008 there are more than 100 projects that range from 1 - $10 \mathrm{~TB}$ of data each. Figure 1 shows the projects with the largest amount of data in NGF. 


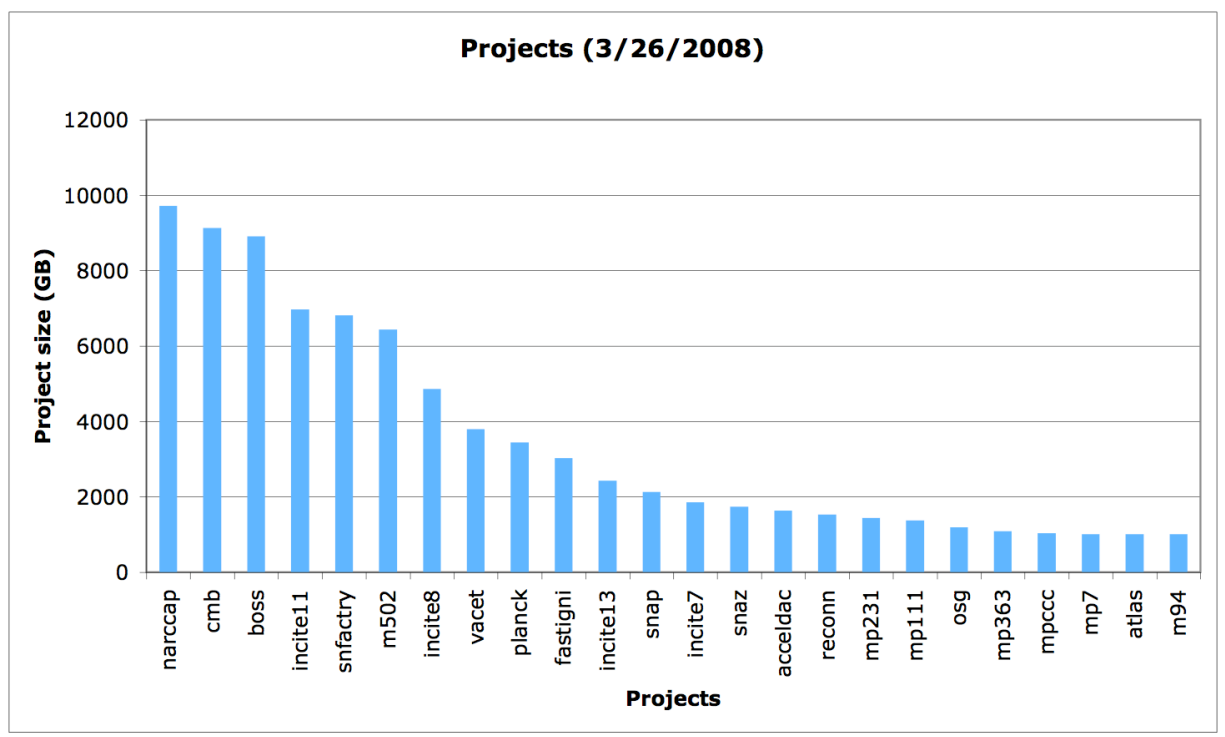

Fig. 1: Projects with data in the NERSC Global File System

The center continually upgrades and adds new hardware to meet users' needs. Figure 2 shows the growth in storage usage for a two-year period, a seven fold increase in data; an average quarterly rate of about $29 \%$. If this trend continues, by 2011 the amount of data in NGF will pass the Petabyte point and if the center continues the existing backup policy, the existing tools and procedures will not be able to meet the backup objectives. We will examine the existing tools and their shortcomings and make recommendations to overcome these challenges.

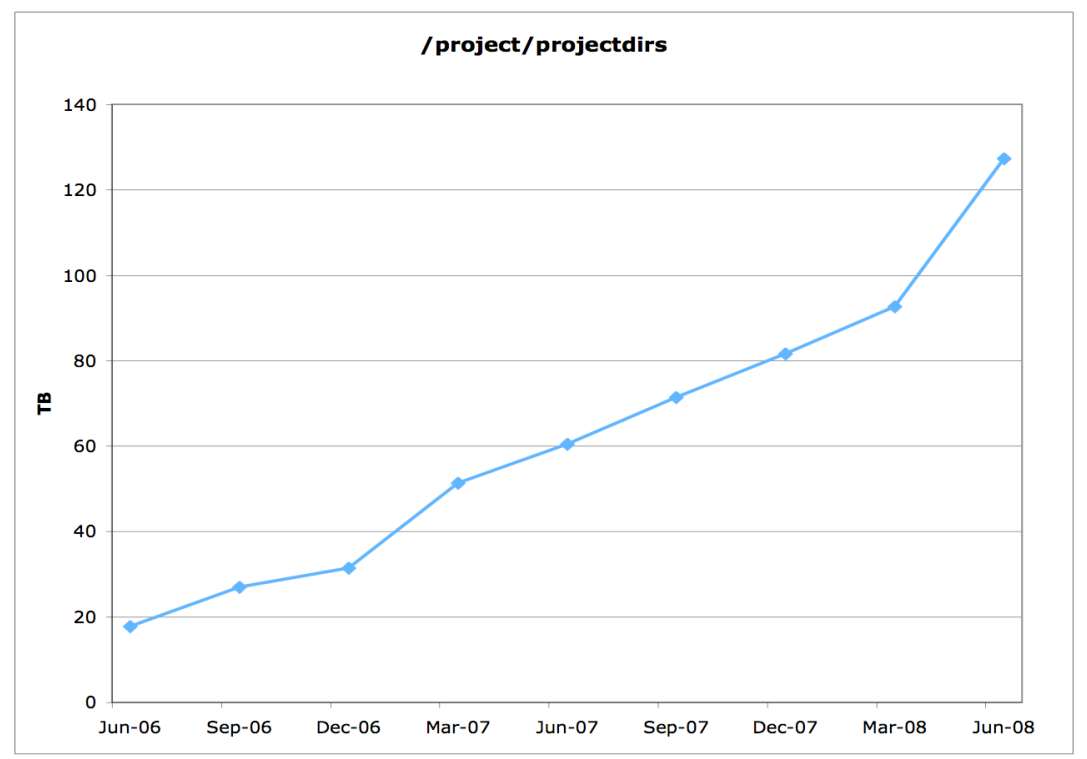

Fig. 2: NGF disk usage growth - two-year period 


\section{Existing tools}

The current NGF backup solution is dependent on the software currently used for the global file system and its configuration. The global file system software is IBM's GPFS version 3.1. Figure 3 shows the current NGF configuration and its components. TSAILUN $^{1}$ is a cluster of servers servicing user's requests. Major characteristics of the system are:

- $230 \mathrm{~TB}$ end user storage with 50+ million inodes accessible from all compute systems (70 TB is allocated to Deep Sky project and is not backed up).

- $24 \mathrm{I} / \mathrm{O}$ server nodes, 2 service nodes

- GPFS 3.1 PTF 20

- DDN 9500 with SATA \& FC, IBM SATA \& FC, Sun SATA, 32 2/4 Gb/s FC

- $5.5 \mathrm{~GB} / \mathrm{s}$ bandwidth for streaming $\mathrm{I} / \mathrm{O}$

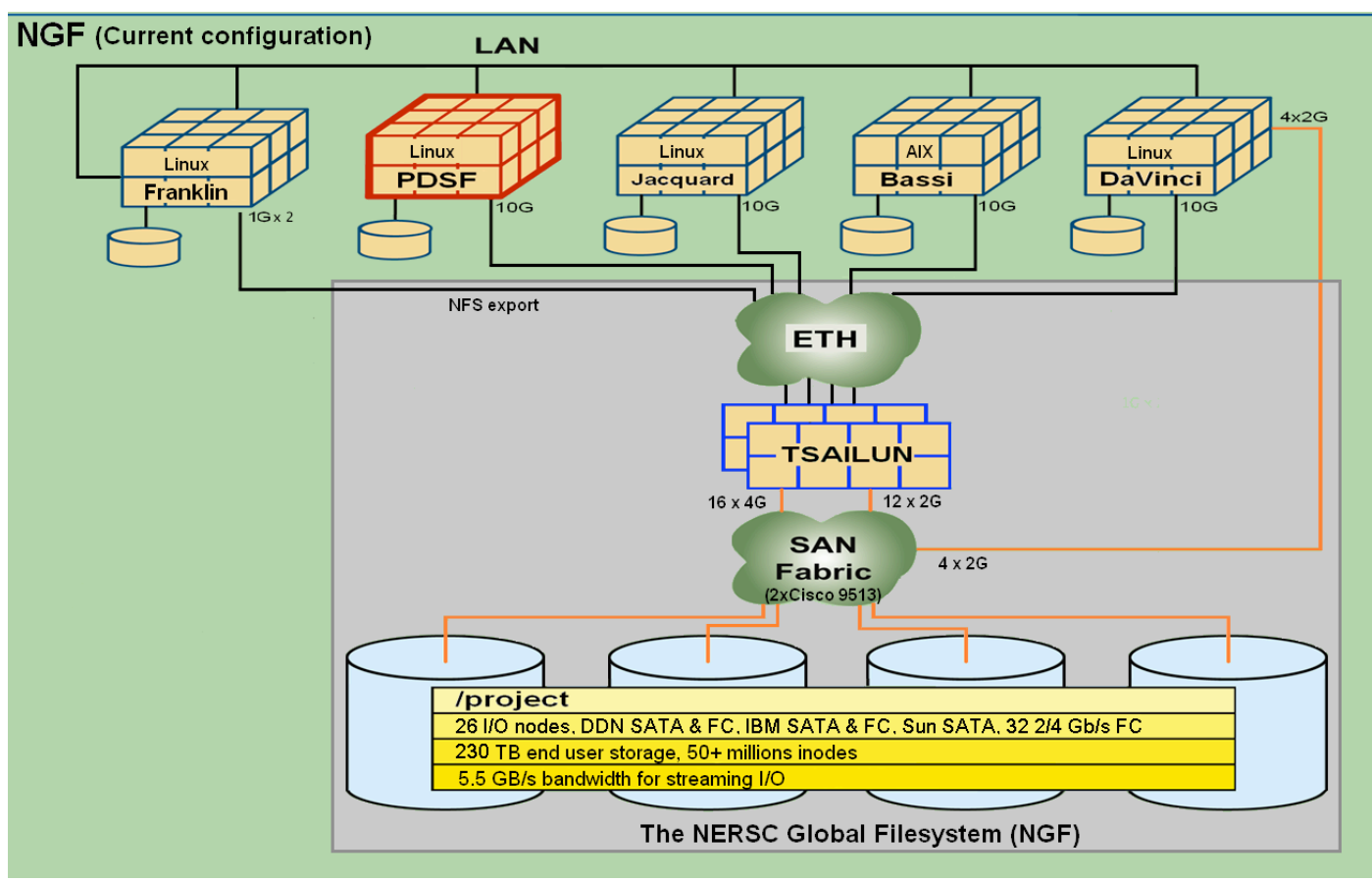

Fig. 3 NGF Configuration as of June 2008

A traditional full backup of the file system is performed periodically using a set of custom scripts. The scripts can be configured to backup a selected set of projects. The backup files are stored in the center's High Performance Storage System (HPSS). A dedicated server in the TSAILUN cluster is used for backup purposes. Initially, a single process was responsible for the whole task but later versions of the script allow for parallel processes to participate in the task. Performing parallel I/O improved the time needed to complete backups significantly.

\footnotetext{
${ }^{1}$ Named after the inventor of the original storage media, paper.
} 


\section{The NGF backup process}

The scripts perform the backup of the "project" area in the following steps:

1. Create a fast scan of 'inodes' using the GPFS native interface

2. Sort the list in descending order by project size

3. A scheduler assigns each project to an agent; there are usually eight agents (processes) for this on a single server. We can have more than one server and more agents.

4. Each agent creates a series of $10 \mathrm{~GB}$ files using 'star' into a specific reserved part of NGF, and sends them to HPSS using HPSS's pftp client.

5. Once an agent finishes its project, the scheduler assigns it a new project from the list.

The fast inode scan generates one record per directory entry containing inode number, file size, file mode, access and modification times, and full file path. This is accomplished in three steps: a) open an inode scan and read the content of each inode, b) read all directory entries starting from a given root directory, and c) merge these records to construct the one record per directory entry. The first two steps use GPFS APIs and the third is to match inode information with file path. It should be noted that the fast inode scan is only useful for incremental backup where a list of changed files can be extracted from the inode scan without costly examination of every file to construct the list.

Figure 4 illustrates the data flow for the above steps. The connection to HPSS has been upgraded to a $10 \mathrm{~Gb}$ network resulting in slightly better transfer rates.

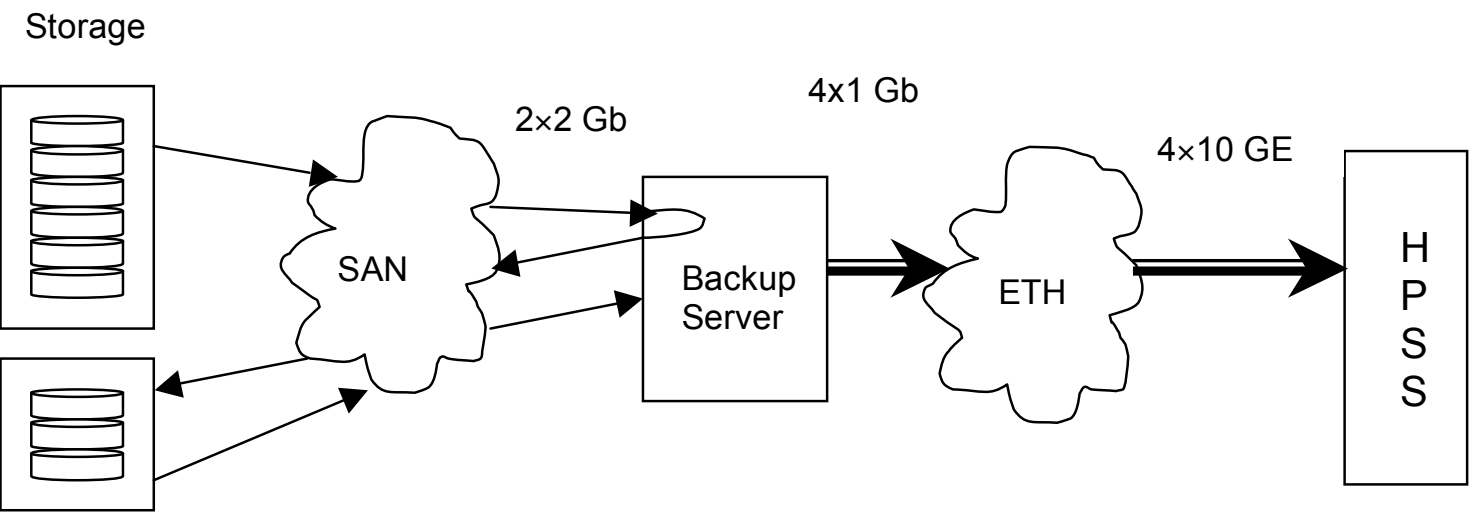

Temp Storage

Fig. 4 Conceptual Data Flow

The times for full backups were extracted from the HPSS daily transfer log files. These files were scanned to get an approximate number of days for each full backup. Figure 5 shows the amount of daily data transferred to HPSS by all clients. The periods of high transfer rates are associated with NGF backup scripts transferring data. 


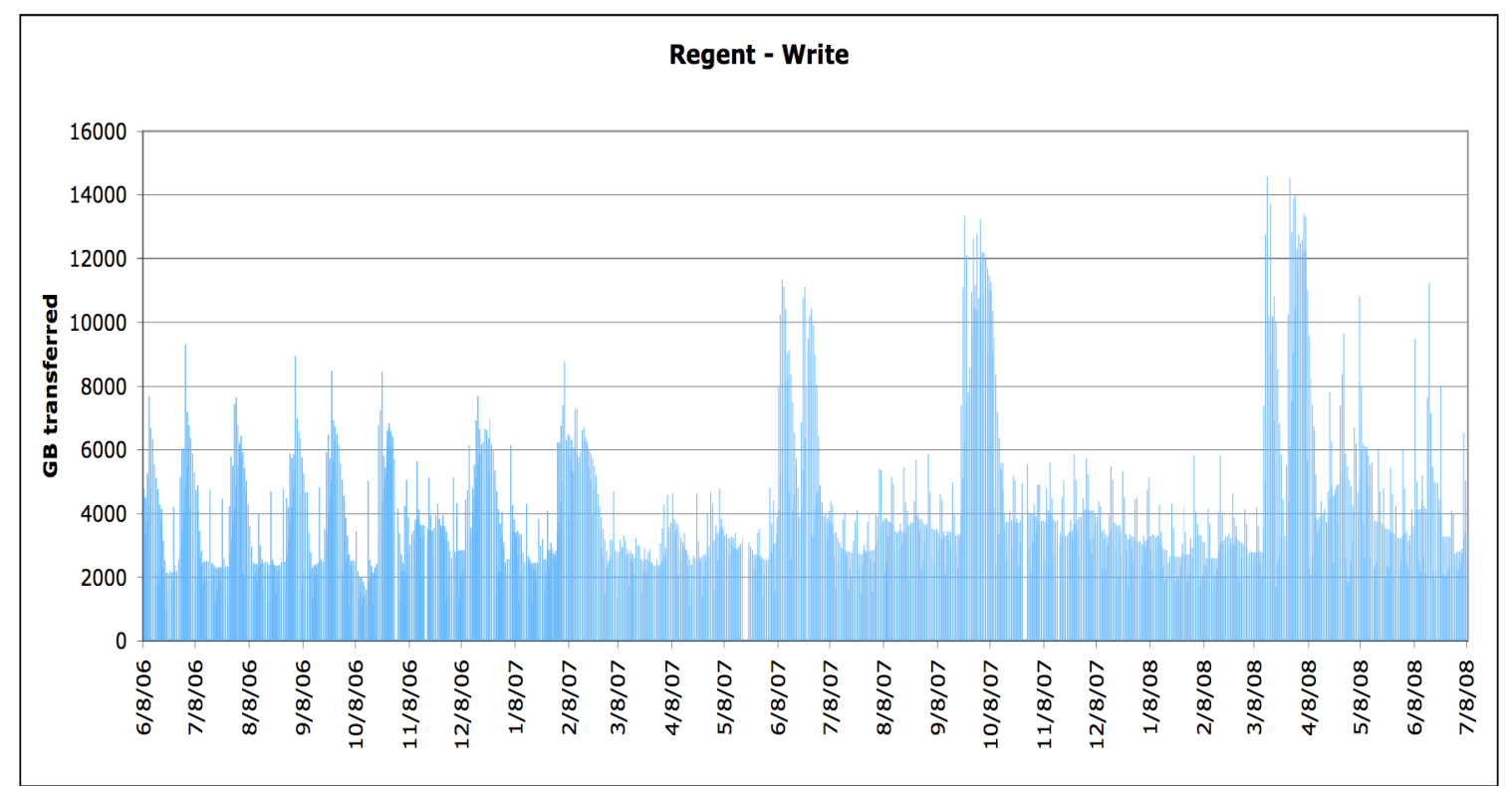

Fig. 5 Daily data transfers to HPSS

Scanning the log files for the NGF backup client yields the data transfers shown in Figure 6 . Note that not all backups were completed successfully and they had to be re-started ${ }^{2}$. In our estimation of time for each backup only the successful runs were considered.

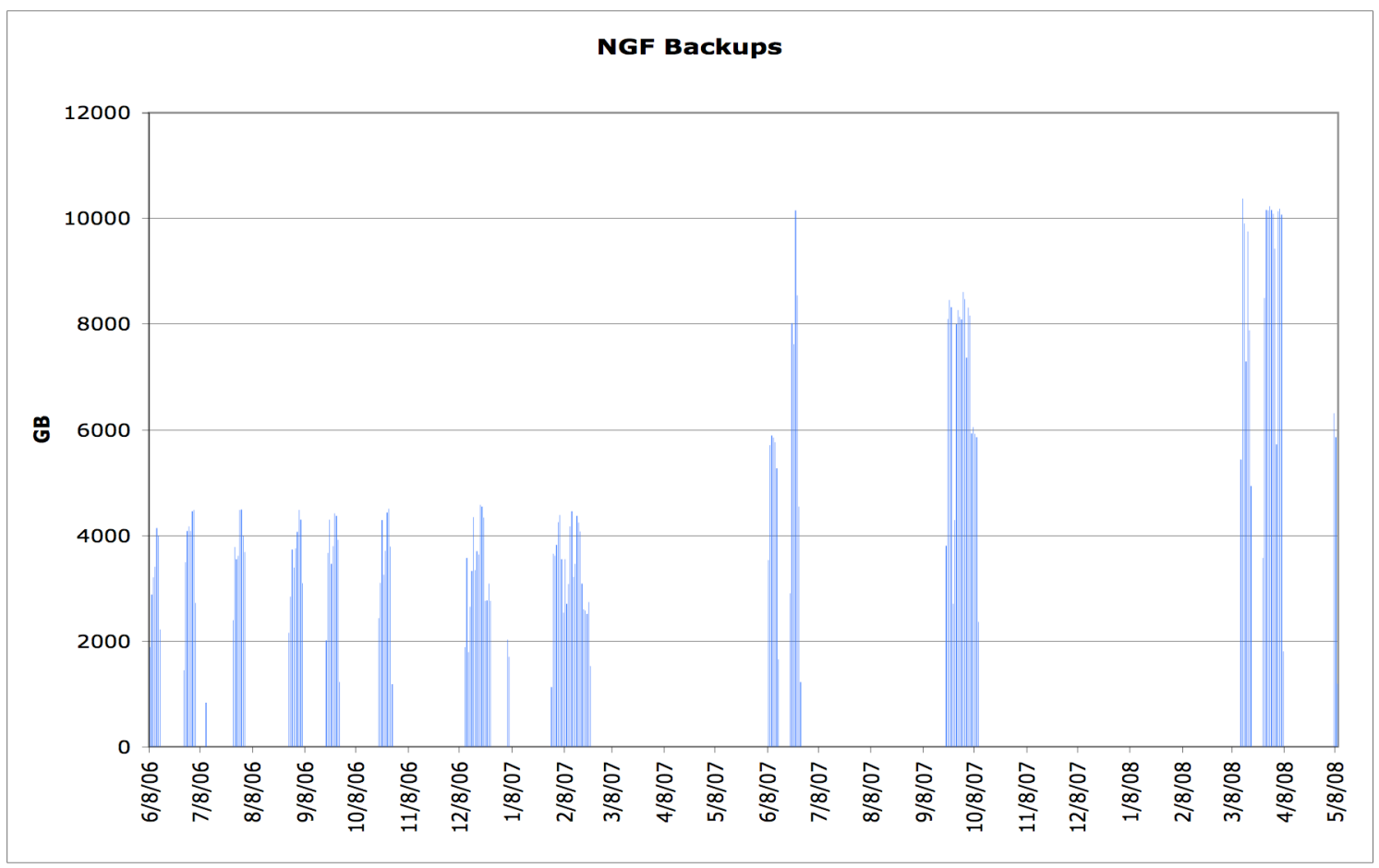

Fig. 6 Daily data transfer to HPSS by backup clients

2 The primary reason for restarting the backup is network and HPSS outage (HPSS has weekly scheduled downtime). Scripts have been improved to handle these outages but at times they fail. 
Figure 7 shows the amount of data backed up and the time, in days, for each. The times include creating the full backup, and transferring that data to HPSS, but not the time it takes for HPSS to migrate the data to tape. The last full backup of $\sim 110 \mathrm{~TB}$ of data took about 13 days to complete. The sharp drop in time for the September 2007 backup is due to increasing the number of processes participating in the process from 1 to 8 , thus parallelizing the task. Each agent is assigned one project at a time and creates a series of $10 \mathrm{~GB}$ files and sends them to HPSS for tape archival using HPSS's Parallel FTP (PFTP) client. It waits until all transfers succeed before getting a new project from the scheduler. The slight improvement in the March 2008 backup is the result of upgrading four HPSS data mover $1 \mathrm{~Gb}$ network connections to $10 \mathrm{~Gb}$.

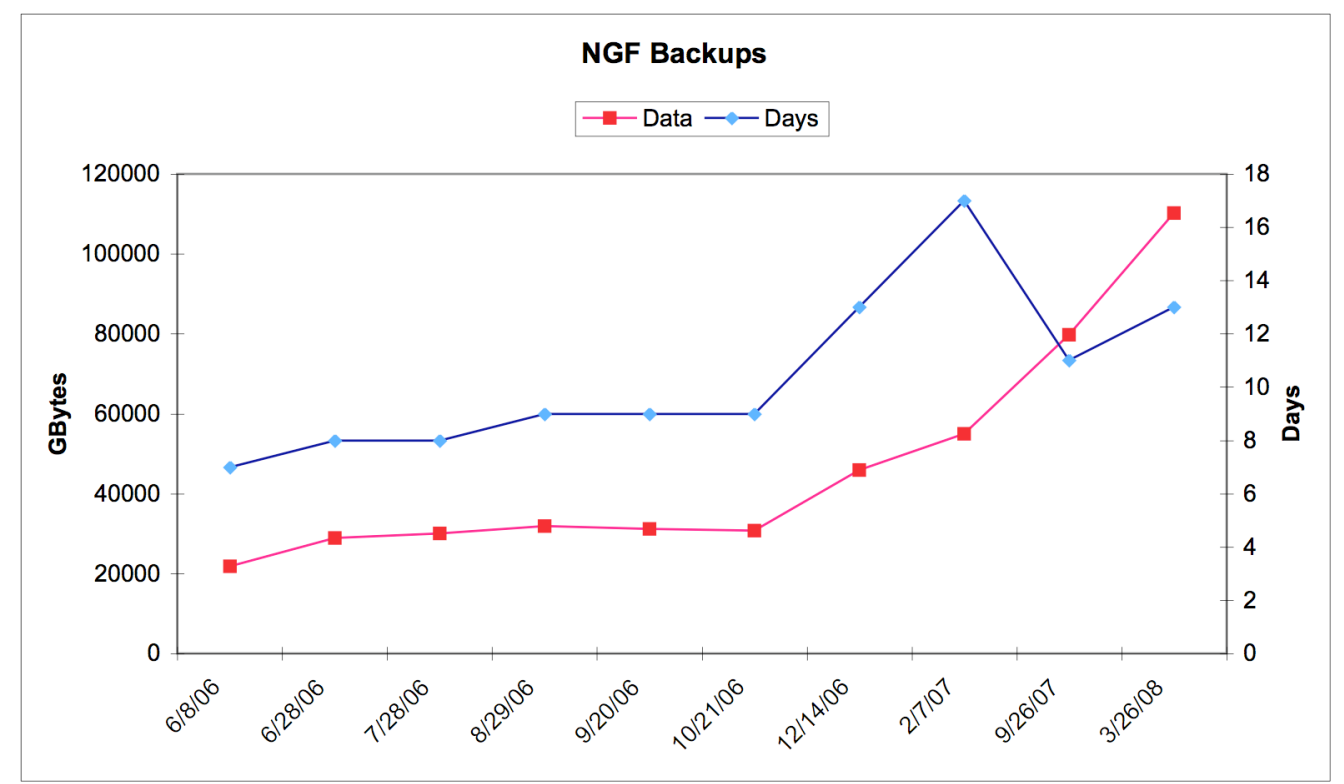

Fig. 7 NGF backups - Two-year Period

It is clear from Figure 7 that the existing tools are not adequate to provide efficient and reliable backup policies for NGF as it continues to grow. There are significant issues currently with the length of time it takes to complete a backup.

\section{Future Improvements}

The single factor having the most impact on the backup process is the communication bandwidth among different (sub)systems participating in the process. Here is a list of specific recommendations for improving the performance:

1. The existing server has two $2 \mathrm{~Gb}$ FC connections to the storage arrays. These connections are used for reading from the main storage arrays, writing the files to temporary storage, and reading them back from the temporary storage for transfer to HPSS. This is the main bottleneck in the present setup (we can get, at best, 200 $\mathrm{MB} / \mathrm{s}$ rate). Eliminating this step by adding direct attached storage to the server should allow higher bandwidth to read files from NGF and speed up the process, assuming that the DAS would have enough bandwidth to avoid contention among processes for write and read. 
2. Adding more servers would also reduce backup time greatly. We estimate that with the present setup we can run the system with 3-4 servers without saturating the rest of the system, excluding the temporary storage spools, with essentially very little impact on the file system. Since all agents share a set of spindles for temporary storage, addition of more servers and agents would saturate this component very quickly. An alternative would be to assign each agent, or group of agents, to different set of disks and minimize the contention.

3. The additional time spent in writing to and reading from the temporary storage can greatly be decreased if solid state disks (SSD) are used inside the server and thus eliminating the extra paths through the SAN fabric. This also allows higher bandwidth to read files from NGF.

4. Each agent waits until the transfer to HPSS is completed before starting on the next set of files. This constitutes approximately $30 \%$ of the total time ${ }^{3}$. HPSS is capable of handling much higher transfer rates. Making the transfer asynchronous can reduce the total time for backup if the system is not always saturated.

5. Consider direct-to-tape transfer in HPSS due to the size of NGF backups.

6. Consider streaming the transfers to HPSS, thus eliminating the step for temporary storage. This, however, requires significant software support from HPSS. The new releases of HPSS, 6.2 and 7.1, have this feature implemented.

7. Figure 8 shows the transfer rates for individual parallel transfers to HPSS during the last full backup. The black points indicate the file segments $(2 \mathrm{~GB})^{4}$ transfer for NGF backup and red points are other system backup clients. The $\mathrm{x}$-axis is the order of each entry in the log files. The vertical lines are transfer log boundaries totaling to $13 \log$ files (days). The NGF backup averages about $50 \mathrm{MB} / \mathrm{sec}$ whereas other clients see rates higher than $200 \mathrm{MB} / \mathrm{sec}$. This is direct consequence of all 8 backup streams sharing the same $\mathrm{FC}$ backend bandwidth ${ }^{5}$. If the average transfer rate can be increased to a higher rate, we can see a rather significant decrease in time for full backup.

\footnotetext{
${ }^{3}$ It is difficult to get an accurate estimate since there are overlaps in operations among agents. A single process backup yielded a $40 \%-45 \%$ of time spend in transfers to HPSS

${ }^{4}$ HPSS PFTP client breaks down files into 2 GB segments for parallel transfers. This is a configurable parameter and can be changed to a higher size

5 The single-stream backup of $10 / 21 / 06$ has a transfer rate of close to $100 \mathrm{MB} / \mathrm{sec}$
} 


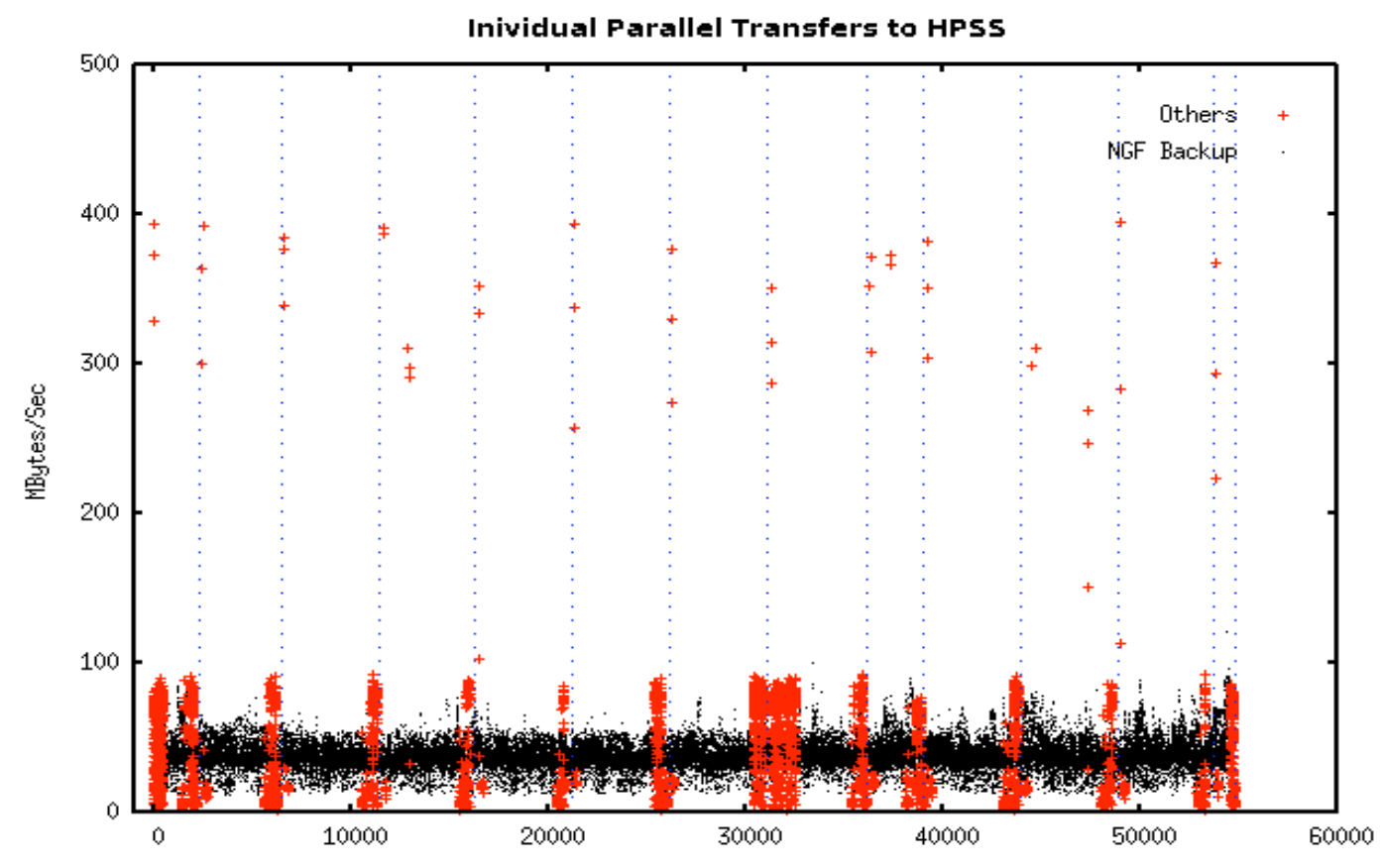

Fig. 8 Individual parallel transfers to HPSS

\section{Shortcomings of existing tools}

- The backup process for NGF relies on the GPFS provided utility for scanning the inodes to create and sort list of files/projects to backup. This utility is relatively fast and it is only useful for incremental backup where a list of modified files can be quickly constructed from inode scan, and it does not have significant impact on full backups. Such an interface might not exist in other file systems.

- Since no locks can be placed on files or directories, users are allowed to modify/delete files before they are actually backed up. This means that the time stamp on the backup does not guarantee that files will be restored to their states indicated by the timestamp.

- No single file can be restored since files in project(s) are tarred up until the file size limit of $10 \mathrm{~GB}$ is reached. One needs to restore all files from beginning of the project to where the requested file is stored, which can take one or more 10GB files to be read.

- No incremental backup can be done with the existing tools.

\section{Conclusions}

We have examined the existing backup utilities at NERSC for NGF full backup. The present trend of $29 \%$ quarterly increase in users data makes the current approach impractical within the next few years. If frequent backups of users' data will be required to restore the global file system to a known state in a Petascale or larger environment, careful consideration should be paid to the requirements and design of tools. A number of steps are outlined that can improve the performance significantly. However, periodic full backup of large file systems will be impractical and will impose a large overhead on 
available resources. A more practical approach is either a selected list of files/directories, determined by users, are backed up, or frequent incremental backup of the file system.

Any backup policy needs to consider the impact on the file system in servicing user's request. Having too many agents participating in the process can impact file system response time, thus degrading performance for end users. Calculations based on the existing $5.5 \mathrm{~GB} / \mathrm{s}$ of NGF streaming with a $5 \%$ overhead on the file system for backup processes give $\sim 250 \mathrm{MB} / \mathrm{s}$ bandwidth, and about 110 hours of backup time for every 100 TB of data.

The backup policy should also consider the value of retaining user data from backups spaced farther apart. Roughly, about $20 \%$ of files have modification date of less than 120 days and if, for instance, a quarterly backup is taken and a disaster occurs requiring restoration of the file system, some user data might render invaluable if they have changed significantly since the last full backup.

Figure 9 shows the time required to transfer $100 \mathrm{~TB}$ of data between any two systems at various bandwidths. Even at a net transfer rate of $250 \mathrm{MB} / \mathrm{s}$ ( $5 \%$ of NGF available bandwidth) between NGF and HPSS, it still takes more than 4.5 days to backup 100 TB of data, not a practical solution for petascale environment.

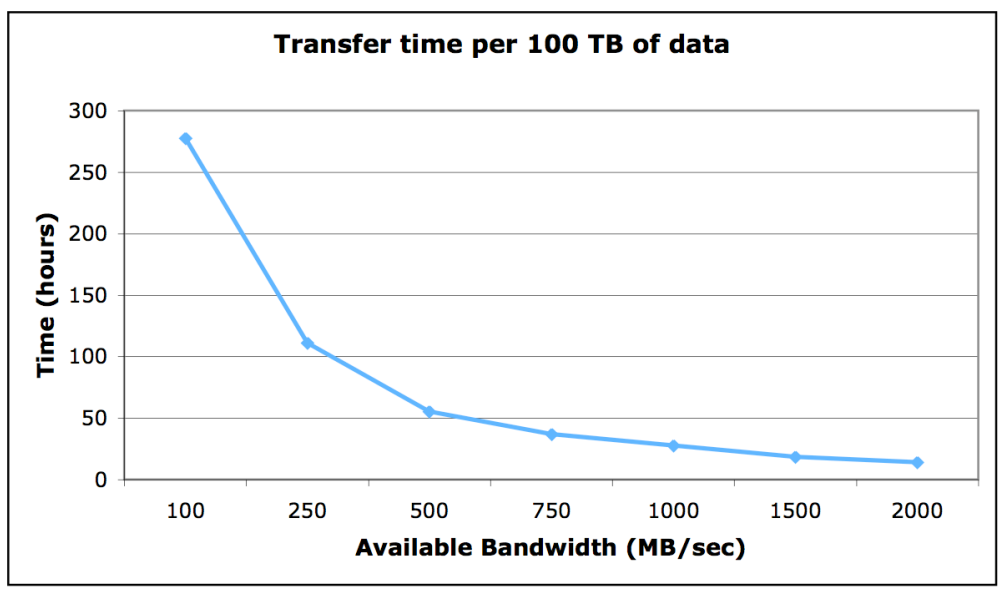

Fig. 9 Transfer time per $100 \mathrm{~TB}$ of data at various bandwidths

\section{Acknowledgment:}

This work was produced by the University of California, Lawrence Berkeley National Laboratory (LBNL) under Contract No. DE-AC02-05CH11231 with DOE. 
